\title{
Dalfampridine extended release tablets: I year of postmarketing safety experience in the US
}

\author{
This article was published in the following Dove Press journal: \\ Neuropsychiatric Disease and Treatment \\ 9 March 2013 \\ Number of times this article has been viewed
}

\section{Michele Jara' \\ Graham Barker ${ }^{2}$ \\ Herbert R Henney 3rd'}

'Acorda Therapeutics, Inc, Ardsley, NY, USA; ${ }^{2}$ Biogen Idec, Inc,

Maidenhead, Berkshire, UK
Correspondence: Michele Jara

Acorda Therapeutics, Inc, 420 Saw Mill

River Road, Ardsley, NY 10502, USA

$\mathrm{Tel}+\mid$ 9|4 3265368

Fax + I 9143474560

Email mjara@acorda.com
Background: Dalfampridine extended release tablets (dalfampridine-ER; prolonged-, modified, or sustained-release fampridine in some countries) were approved in the US to improve walking in patients with multiple sclerosis, as demonstrated by improvement in walking speed. Postmarketing safety experience is available from exposure of approximately 46,000 patients in the US from product approval through March 2011.

Objective: To provide a descriptive analysis of all spontaneously reported postmarketing adverse events (AEs) for dalfampridine-ER since product launch.

Methods: AE data were extracted from the safety database from product launch through March 31, 2011; AEs were classified using the Medical Dictionary for Regulatory Activities. Seizure cases were reviewed for patient demographics, time to event from treatment onset, and presence of additional risk factors.

Results: The most frequently reported postmarketing AEs were similar to those reported during clinical development: dizziness, insomnia, balance disorder, headache, nausea, urinary tract infection, asthenia, and back pain (all included in US product labeling). New clinically significant findings are related to lack of efficacy and inappropriate dosing. Of the approximately 46,000 patients exposed, 85 seizures were reported ( 5.4/1000 patient-years), of which 82 were reported or confirmed by a health care practitioner ( $~ 5.2 / 1000$ patient-years). Beyond the intrinsic multiple sclerosis-related seizure risk, more than half of the 85 cases $(62 \%)$ had an additional potential risk factor for seizure including a previous history of convulsions, renal impairment, incorrect dosing, or use of concurrent medications with a labeled seizure risk. Duration of treatment prior to the seizure ranged from one dose to 365 days; 26/85 (31\%) patients suffered a seizure within a week of starting treatment.

Conclusion: Spontaneous safety data from the US postmarketing experience were consistent with the safety profile seen during clinical development. Although first-year seizure incidence was not substantially different from that observed in dalfampridine-ER clinical trials, patients should be monitored for concomitant use of drugs that lower seizure threshold.

Keywords: dalfampridine, fampridine, multiple sclerosis, adverse events, postmarketing safety, seizure

\section{Introduction}

Dalfampridine extended release tablets (dalfampridine-ER tablets), $10 \mathrm{mg}$ taken 12 hours apart, were approved by the US Food and Drug Administration to improve walking in patients with multiple sclerosis (MS). ${ }^{1}$ It has been available in the US since March 2010, and was also approved in the European Union in July 2012, where it is known as prolonged-release fampridine, and in other countries, where it is known as modified or sustained-release fampridine. Approval was based on a Phase II and 
two Phase III clinical trials that demonstrated an increase in walking speed using the Timed 25-Foot Walk. ${ }^{2-4}$

Dalfampridine-ER is the chemical entity 4-aminopyridine (4-AP), a voltage-dependent potassium channel blocker with a proposed mechanism of action in patients with MS of restoring conduction in demyelinated axons. ${ }^{5}$ Early studies showed that the narrow therapeutic range of 4-AP in immediate-release formulations that required three to four times daily dosing resulted in an incidence of adverse events (AEs), including a risk for seizures, that was dependent on plasma concentrations. ${ }^{6-8}$ These observations suggested the need for, and the development of, the dalfampridine-ER formulation to reduce the risk of toxicity and decrease dosing frequency.

In the three clinical trials, dalfampridine-ER was observed to have a favorable tolerability profile at the recommended dosage of a single 10-mg tablet taken twice daily at approximately 12 hours apart. The incidence of AEs was similar among the trials, and AEs were generally of mild or moderate severity. ${ }^{2-4}$ The most commonly reported AEs were balance problems, paresthesia, dizziness, anxiety, insomnia, and confusion, and these can be ascribed to the stimulatory effects of dalfampridine-ER on the nervous system.

Dalfampridine-ER is known to have a dose-dependent risk of seizure, ${ }^{6,9}$ and therefore seizure-related events were of special interest during the registration trials. Seizures were reported in $0.2 \%$ of patients during the three clinical trials, and in the pooled extensions of these trials, with patient exposure to dalfampridine-ER of up to 5 years, the seizure rate was $4.1 / 1000$ patient-years (95\% confidence interval [CI] 1.3/1000-9.6/1000 patient-years). ${ }^{1}$ However, it is also recognized that MS patients have an approximately three-fold higher risk of seizure than that of the general population. ${ }^{10,11}$ Although the estimates of seizure incidence in MS patients vary, the most relevant estimate is likely from Eriksson et al, ${ }^{12}$ since they estimated the incidence of first seizure. Their estimate of 3.49/1000 patient-years (95\% CI 1.96/1000-5.02/1000 patient-years) in a Swedish MS population was similar to the rate reported in the pooled extension studies. Although dalfampridine-ER is contraindicated in patients with a history of seizures, ${ }^{1}$ a Risk Evaluation and Mitigation Strategy (REMS) was instituted to inform health care providers and patients about seizure risk with use of dalfampridine-ER. ${ }^{13}$

While information from clinical trials can be a useful indicator of expected tolerability, such data are not necessarily representative of what may be observed in the clinical practice setting. Thus, postmarketing surveillance has become an integral component of monitoring drug safety. The current study was undertaken to provide an analysis of the postmarketing safety of dalfampridine-ER in the US based on spontaneously reported AEs from product launch through March 2011, encompassing the first year of marketing. Furthermore, data from the postapproval program of enhanced surveillance and assessment were evaluated to further characterize the seizure risk potential.

\section{Material and methods}

In the US, health care professionals and consumers can report AEs to a product's manufacturer, who is then required to send all cases to the Food and Drug Administration as specified by regulations. ${ }^{14}$ This prospectively designed evaluation was performed to provide a descriptive analysis of all postmarketing dalfampridine-ER spontaneously reported AEs regardless of causality in the manufacturer's product safety database from March 1, 2010 through March 31, 2011. The product safety database is an Oracle Argus Safety AE management system (Oracle Corporation, Redwood Shores, CA, USA) with all reported AEs entered into the system and processed through a vendor (United BioSource Corporation, Chevy Chase, MD, USA). All AEs in the database were classified using the internationally recognized Medical Dictionary for Regulatory Activities (MedDRA; version 13.0). The proportion of $A E$ reports was estimated by event, as classified at the MedDRA preferred term level. Commonly reported AEs were defined as those with a prevalence $\geq 2 \%$ of all reported AEs.

Additional analysis was conducted among the patients taking dalfampridine-ER to determine the incidence of reported seizure. Dalfampridine-ER is dispensed via a closed distribution system that provides information on the number of patients exposed and enables estimates of patient-years of exposure based on the number of bottles distributed. The incidence rate of seizure was calculated using the estimate of person-time of exposure of 15,850 person-years based on data from the closed distribution system. All confirmed and potential seizure reports were subject to detailed and proactive follow-up using a structured seizure questionnaire designed to collect information on dose, regimen, usage, seizure history, concomitant medications, and any prior use of pharmacy-compounded formulations. Additional information on seizure cases was extracted from a case list kept as part of this enhanced pharmacovigilance system. Each seizure case was reviewed to ascertain patient demographics, time to event from treatment onset, and whether additional risk factors for seizure were present. Seizures reported or 
confirmed by a health care practitioner were considered confirmed seizures, and those reported only by a consumer were considered potential seizures. Any case reported with an event classified under the MedDRA High-Level Group Term Seizures (including subtypes) was considered a seizure.

AEs for pharmacy-compounded 4-AP reported to Acorda Therapeutics, Inc (Ardsley, NY, USA), the manufacturer of dalfampridine-ER, were also entered into the Argus Safety database. The safety database was queried to determine the number of seizure reports out of the total number of AE reports for compounded 4-AP. As above, any case reported with an event classified under the MedDRA High-Level Group Term Seizures (including subtypes) was considered a seizure.

\section{Results}

Data on the postmarketing safety experience were available from the exposure of approximately 46,000 patients who filled a prescription for dalfampridine-ER during the first year of product availability. This patient exposure represents approximately 15,850 patient-years. Demographic data were available for 44,114 patients (95.5\%). Gender distribution was similar between the clinical trial and postmarketing populations, with approximately $70 \%$ women in both populations. Overall, the age distribution of the postmarketing population was similar to that in the clinical trials. The mean age of the postmarketing population, 54.8 years, was older than that of the clinical trial population, 51.7 years. However, the postmarketing population had a wider age range, 14-101 years, compared with 25-73 years in the clinical trial population, and a higher proportion of patients aged $\geq 65$ years, $18 \%$ compared with $6 \%$ in the clinical trial population.

During the first year of dalfampridine-ER use, a total of 11,549 AEs were reported in the postmarketing population, and the most frequently reported AEs are shown in Table 1. As also shown in Table 1, the majority of these AEs were listed in the package insert including dizziness, insomnia, balance disorder, headache, nausea, urinary tract infection, asthenia, and back pain. New findings from this uncontrolled population were related to inappropriate dosing reported as an AE ( $\mathrm{n}=573)$, defined as an event code of "inappropriate schedule of dose administration" when reports related to lack of efficacy were received in cases of dosing less than the approved daily dose. Patients who were reported with a double dose of drug ingested, or when the dosing interval was less than 8 hours, were assigned event codes of accidental overdose $(n=36)$, intentional overdose $(n=1)$, or overdose $(\mathrm{n}=5)$, depending on individual case information.
Table I Most frequently reported adverse events, defined as having a prevalence of $\geq 2 \%$ of all reported events $(n=11,549)$, by Medical Dictionary for Regulatory Activities preferred term in the postmarketing population through March 31, 201 I

\begin{tabular}{lll}
\hline $\begin{array}{l}\text { Medical Dictionary for Regulatory } \\
\text { Activities preferred term }\end{array}$ & $\begin{array}{l}\mathbf{n} \text { (\% of total } \\
\text { events) }\end{array}$ & $\begin{array}{l}\text { Event } \\
\text { status }\end{array}$ \\
\hline Dizziness & $658(5.7)$ & Labeled \\
Insomnia & $524(4.5)$ & Labeled \\
Balance disorder & $450(3.9)$ & Labeled \\
Headache & $375(3.2)$ & Labeled \\
Nausea & $323(2.8)$ & Labeled \\
Urinary tract infection & $276(2.4)$ & Labeled \\
Asthenia & $235(2.0)$ & Labeled \\
Back pain & $232(2.0)$ & Labeled \\
Condition aggravated & $347(3.0)$ & Not listed \\
Gait disturbance & $239(2.1)$ & Not listed \\
Drug ineffective & $517(4.5)$ & Expected \\
Inappropriate schedule of drug & $573(5.0)$ & Not listed \\
administration & & \\
\hline
\end{tabular}

During the first year, a total of 85 cases of seizure were reported, 82 of which were either reported or confirmed by a health care practitioner. Of the 85 cases, three seizure cases were reported directly by patients but were not confirmed by a health care practitioner. More than $96 \%$ of the seizures $(82 / 85)$ were tonic-clonic convulsions. Among the approximately 46,000 patients exposed, 85 seizure cases equate to a rate of approximately 5.4 cases per 1000 patient-years of use. Subtracting the three unconfirmed cases lowers the estimated incidence rate to approximately 5.2 cases per 1000 patientyears of use.

The demographics of patients with reported seizure were reflective of the overall postmarketing population; $78 \%$ of the reported cases were in women (66 women, 19 men) and the mean patient age was 53 years (range 15-76 years), with three cases missing age information. Duration of treatment prior to the event ranged from one dose to 365 days; 26/85 (31\%) patients experienced a seizure within a week of starting treatment. After the first week of treatment, the incidence rate of reported seizures was approximately 3.9/1000 patient-years of use.

Of the reported seizures, $62 \%(53 / 85)$ had at least one additional risk factor for seizure, with 55\% (47/85) having one other risk factor and $7 \%$ having two or three other risk factors. Risk factors included previous history of seizure, renal impairment, dosing error of either double dose or dosing interval less than 12 hours, use of concurrent medications known to lower seizure threshold, and head injury with a subdural hematoma prior to the event. Among the patients experiencing a seizure on dalfampridine-ER, 53\% had concomitant use of one or more medications known to 
lower seizure threshold (45/85; Table 2) including baclofen in $33 \%$ of reported cases, amantadine in $22 \%$, bupropion in $8 \%$, oxybutynin in $8 \%$, and tramadol in $4 \%$ (Table 3 ). The proportion of patients experiencing a seizure with concomitant use of medications known to lower seizure threshold was lower than the proportion observed using these medications in the overall clinical trial patient population. Other risk factors reported in patients experiencing a seizure on dalfampridine-ER included a history of seizure in five of the reported cases (6\%), some level of renal impairment qualitatively reported in three cases (4\%), an associated dosing error in five cases $(6 \%)$, and two cases with head injury with subdural hematoma ( $2 \%$; Table 2$)$. In one of the cases of head injury, the injury occurred approximately 5 months prior to the seizure and the patient had been on dalfampridine-ER for 2 weeks at the time of the seizure. In the other case, the head injury was sustained immediately prior to the seizure and was more likely to have had a causal contribution.

Of 106 unsolicited AEs received by Acorda for compounded formulations of 4-AP during the same time period, $7.5 \%(n=8)$ were seizures compared with $0.7 \%$ for dalfampridine-ER (85/11,549 AEs were seizures) reported in the same time period. Of the reported seizures in patients using compounded 4-AP, more than half of the reported cases were in men (four men, three women), and the mean patient age was 50 years (range 14-79 years), with one case missing both gender and age information. An additional risk factor for seizure was reported in $37.5 \%$ of patients experiencing a seizure on compounded 4-AP, including a history of seizure in one of the reported seizures (12.5\%), an associated dosing error in one case (12.5\%), and concomitant use of medications known to lower seizure threshold, both amantadine and bupropion, in one case (12.5\%).

Besides seizure, other medically serious AEs that were reported in the first year of marketing included cardiac arrest $(n=2)$, cardiac failure $(n=3)$, myocardial infarction $(n=3)$, hepatic failure $(n=1)$, hepatitis $(n=1)$, ischemia $(n=1)$, pancreatitis $(n=1)$, renal failure $(n=2)$, respiratory failure $(n=2)$, and sepsis $(n=1)$. There were twelve events related to suicidal thoughts and actions, including the MedDRA terms completed suicide $(\mathrm{n}=1)$, suicide attempt $(n=1)$, suicidal behavior $(n=1)$, and suicidal ideation $(n=9)$. Of these cases, five had a history of depression with three using concomitant antidepressants. Of the seven remaining patients, five had missing information on medical history or use of concomitant medications (four were missing both and one was missing information on concomitant medication use). In the first year of marketing, there was a report of one patient with a symptom complex resembling anaphylaxis, although anaphylaxis was not specifically diagnosed.

\section{Discussion}

The results of this first postmarketing evaluation of dalfampridine-ER since product approval show that AEs reported among the patients with MS treated with this drug in the clinical setting were relatively consistent with expectations based on clinical trials. The most frequently reported AEs in the postmarketing population were those that have previously been identified as common events in the clinical trials. These AEs including dizziness, insomnia, balance disorder, headache, nausea, urinary tract infection, asthenia, and back pain are already detailed in the US product labeling, ${ }^{1}$ and although causality was not determined in the current evaluation, it is likely that the occurrence of at least some of these events may be related to the stimulatory effects of dalfampridine-ER on the nervous system.

While "condition aggravated" and "gait disturbance" were frequently reported as postmarketing AEs, neither of these events was reported more frequently in dalfampridine-ER patients relative to placebo in clinical trials. Reports related to lack of efficacy were received, and were listed as AEs. However, lack of efficacy may be expected with any symptomatic therapy, and since dalfampridine-ER in particular was effective in $35 \%-43 \%$ of users in the Phase III clinical trials, 3,4 the lack of effectiveness in a proportion of treated patients was not surprising. In contrast, it was surprising that reports

Table 2 Patients with single and multiple confounding factors for seizure among patients experiencing seizure during postmarketing surveillance of dalfampridine extended release tablets ${ }^{\mathrm{a}}$

\begin{tabular}{|c|c|c|c|c|c|}
\hline & $\begin{array}{l}\text { Concomitant } \\
\text { medications }\end{array}$ & $\begin{array}{l}\text { Seizure } \\
\text { history }\end{array}$ & $\begin{array}{l}\text { Renal } \\
\text { impairment }\end{array}$ & $\begin{array}{l}\text { Dosing } \\
\text { errors }\end{array}$ & Total, n (\%) \\
\hline Concomitant medications & $39^{b}$ & 2 & 2 & 3 & $45(53)^{c}$ \\
\hline Seizure history & 2 & 3 & $\mathrm{I}$ & 0 & $5(6)^{c}$ \\
\hline Renal impairment & 2 & I & $\mathbf{I}$ & 0 & $3(4)^{c}$ \\
\hline Dosing errors & 3 & 0 & 0 & 2 & $5(6)$ \\
\hline
\end{tabular}

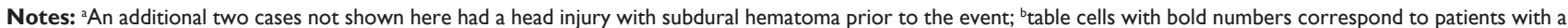
single confounding factor; 'one case met three criteria for confounding factors: epileptogenic concomitant medication use, seizure history, and renal impairment. 
Table 3 Types of medications known to lower seizure threshold used concurrently with dalfampridine extended release tablets among reported seizure cases

\begin{tabular}{lll}
\hline Concomitant medication & \multicolumn{2}{l}{$\begin{array}{l}\text { Total seizure cases } \\
(\mathbf{n}=\mathbf{8 5})\end{array}$} \\
\cline { 2 - 3 } & $\mathbf{n}$ & $\%$ \\
\hline Amantadine & 19 & 22.4 \\
Baclofen & 28 & 32.9 \\
Bupropion & 7 & 8.2 \\
Oxybutynin & 7 & 8.2 \\
Tramadol & 3 & 3.5 \\
\hline
\end{tabular}

of inappropriate dosing were higher than expected ( $6 \%$ of all AEs) given that there is only one approved dose.

In the first year of marketing, the overall incidence rate of seizure was 5.4/1000 patient-years of use including the three unconfirmed patient-reported seizures, and approximately one-third of the cases occurred within the first week of exposure. After the first week of treatment, the incidence rate was 3.9/1000 patient-years of use. These observed seizure rates are comparable to the incidence rate from the long-term open-label trials at 4.1/1000 patient-years (95\% CI 1.3/1000-9.6/1000 patient-years). ${ }^{1}$ For perspective, it should again be noted that the risk of seizure among individuals with MS is three-fold higher than that of the general population, ${ }^{10,11}$ with an estimated incidence of first seizure of 3.49/1000 patient-years (95\% CI 1.96/1000-5.02/1000 patient-years). ${ }^{12}$

Of additional clinical relevance is the observation that $62 \%$ of the dalfampridine-ER patients reported with seizure had one or more other risk factors for seizure in addition to the seizure risk already inherent in people with MS..$^{15,16}$ Of note, five of the patients with seizures had a seizure history, and three had renal impairment; a history of either of these medical conditions is a contraindication for use of dalfampridine-ER. ${ }^{1}$ Furthermore, five of the seizures were in patients reported as having dosing errors, including intervals shorter than 8 hours. While it is important to adhere to the prescribing guidelines as well as the approved dosing schedule for dalfampridine-ER, these results suggest that patients may either be inadequately evaluated for the presence of contraindications or are treated despite the presence of contraindications. Such a suggestion has previously been reported in a study that observed that at the time of dalfampridine-ER prescription, $3 \%$ and $5 \%$ of users had a history of seizure or some level of renal impairment, respectively. ${ }^{17}$ Although use of medications reducing the seizure threshold is not a specific contraindication, the fact that slightly more than half of the patients experiencing a seizure $(53 \%)$ had concurrent use of one or more medications known to lower seizure threshold, highlights the importance of careful monitoring for use of such medications.

Medically significant AEs were infrequently reported in the first year of dalfampridine-ER use and did not seem to show a pattern indicating a new safety issue. Reports of suicidal behavior and actions may be related to the elevated risk of suicidal thoughts and behaviors in the MS population, which may explain the cases seen in the postmarketing database. A high lifetime prevalence of depression in MS patients increases the risk of suicidal ideation and behavior, and the risk of completed suicide has been reported to be as much as 7.5 times higher than that of the general population. ${ }^{18}$ AEs related to suicide were not seen in the placebo-controlled clinical trials for dalfampridine-ER.

Interpretation of the results of this postmarketing study should take into account the limitations of the study, including that all AEs were evaluated regardless of causality, rather than adverse drug events that may be specifically related to utilization of dalfampridine-ER. Additionally, since these AEs were dependent on spontaneous reporting, it is possible that some events may have been underreported.

\section{Conclusion}

Spontaneous safety data emerging from the US postmarketing experience of dalfampridine-ER are consistent in terms of types of events with the safety profile seen during clinical development. During the initial marketing period, inappropriate dosing and lack of efficacy were also reported. First-year seizure incidence was not substantially different from the incidence observed in long-term open-label trials of dalfampridine-ER. A high percentage of dalfampridine-ER users reported with seizure were also using medications known to lower seizure threshold. Although these rates were lower than would be predicted from the clinical trial population, it is nevertheless advisable that prudent clinical judgment be used when prescribing dalfampridine-ER when taken with drugs that lower seizure threshold. These data suggest that the favorable safety and tolerability profile observed in clinical trials extends to the clinical setting. However, since this drug is likely to be used by patients for extended periods of time, longer-term postmarketing surveillance will be necessary to further characterize the clinical safety profile of dalfampridine-ER.

\section{Acknowledgments}

This study was funded by Acorda Therapeutics, Inc. Editorial assistance in preparation of this manuscript was provided by 


\section{E. Jay Bienen of The Curry Rockefeller Group, LLC. Editorial} assistance was funded by Acorda Therapeutics, Inc.

\section{Disclosure}

Michele Jara and Herbert R Henney 3rd are employees of Acorda Therapeutics, Inc, and hold stock in the company. Graham Barker is currently an employee of Mitsubishi Pharma Europe Ltd; however, he was an employee of Biogen Idec, Inc, at the start of development of this paper. The authors confirm that this paper is an accurate representation of the study results.

\section{References}

1. Ampyra ${ }^{\circledR}$ (dalfampridine extended release tablets) [prescribing information]. Ardsley, NY: Acorda Therapeutics, Inc; 2012.

2. Goodman AD, Brown TR, Cohen JA, et al; Fampridine MS-F202 Study Group. Dose comparison trial of sustained-release fampridine in multiple sclerosis. Neurology. 2008;71(15):1134-1141.

3. Goodman AD, Brown TR, Krupp L, et al; Fampridine MS-F203 Investigators. Sustained-release oral fampridine in multiple sclerosis: a randomised, double-blind, controlled trial. Lancet. 2009; 373(9665):732-738.

4. Goodman AD, Brown TR, Edwards KR, et al; MSF204 Investigators. A phase 3 trial of extended release oral dalfampridine in multiple sclerosis. Ann Neurol. 2010;68(4):494-502.

5. Dunn J, Blight A. Dalfampridine: a brief review of its mechanism of action and efficacy as a treatment to improve walking in patients with multiple sclerosis. Curr Med Res Opin. 2011;27(7):1415-1423.

6. Haut SR, Bienen EJ, Miller A. Clinical overview of the seizure risk of dalfampridine. Expert Opin Drug Saf. 2012;11(4):651-657.

7. Bever CT Jr, Young D, Anderson PA, et al. The effects of 4-aminopyridine in multiple sclerosis patients: results of a randomized, placebo-controlled, double-blind, concentration-controlled, crossover trial. Neurology. 1994; 44(6):1054-1059.
8. Van Diemen HA, Polman CH, Koetsier JC, Van Loenen AC, Nauta JJ, Bertelsmann FW. 4-aminopyridine in patients with multiple sclerosis: dosage and serum level related to efficacy and safety. Clin Neuropharmacol. 1993;16(3):195-204.

9. Cornblath DR, Bienen EJ, Blight AR. The safety profile of dalfampridine extended release in multiple sclerosis clinical trials. Clin Ther. 2012;34(5):1056-1069.

10. Nicoletti A, Sofia V, Biondi R, et al. Epilepsy and multiple sclerosis in Sicily: a population-based study. Epilepsia. 2003;44(11):1445-1448.

11. Poser CM, Brinar VV. Epilepsy and multiple sclerosis. Epilepsy Behav. 2003;4(1):6-12.

12. Eriksson M, Ben-Menachem E, Andersen O. Epileptic seizures, cranial neuralgias and paroxysmal symptoms in remitting and progressive multiple sclerosis. Mult Scler. 2002;8(6):495-499.

13. Acorda Therapeutics, Inc. Ampyra (dalfampridine) Extended Release Tablets. Risk Evaluation and Mitigation Strategy. Ardsley, NY: Acorda Therapeutics; 2010. Available from: http://www.accessdata. fda.gov/drugsatfda docs/label/2010/022250s000REMS.pdf. Accessed November 20, 2012

14. US Food and Drug Administration. FDA Adverse Event Reporting System (FAERS) (formerly AERS). Updated September 10, 2012. Available from: http://www.fda.gov/Drugs/ GuidanceComplianceRegulatoryInformation/Surveillance/ AdverseDrugEffects/. Accessed December 5, 2012

15. Koch M, Uyttenboogaart M, Polman S, De Keyser J. Seizures in multiple sclerosis. Epilepsia. 2008;49(6):948-953.

16. Kelley BJ, Rodriguez M. Seizures in patients with multiple sclerosis: epidemiology, pathophysiology and management. CNS Drugs. 2009;23(10):805-815.

17. Jara M, Sidovar M, Henney HR 3rd. Prescriber utilization study of dalfampridine extended release tablets [abstract]. Int J MS Care. 2012;14(Suppl 2):34.

18. Siegert RJ, Abernethy DA. Depression in multiple sclerosis: a review. J Neurol Neurosurg Psychiatry. 2005;76(4):469-475.
Neuropsychiatric Disease and Treatment

\section{Publish your work in this journal}

Neuropsychiatric Disease and Treatment is an international, peerreviewed journal of clinical therapeutics and pharmacology focusing on concise rapid reporting of clinical or pre-clinical studies on a range of neuropsychiatric and neurological disorders. This journal is indexed on PubMed Central, the 'PsycINFO' database and CAS.

\section{Dovepress}

The manuscript management system is completely online and includes a very quick and fair peer-review system, which is all easy to use. Visit http://www.dovepress.com/testimonials.php to read real quotes from published authors. 